management and peace building lie in the very nature of the international crisis management system itself. As long as we will not get closer to common integrated crisis management or peace-building concepts and strategies, based on at least a minimal political consensus of the representatives of the concerned country and major international donors about the intended end states and how to get there, real progress is unlikely. Deficits in accounting for the complex securitydevelopment nexus when engaging in peace building are - at the national level - mainly due to political conflicts of interest between departments and/or political parties, to the structural inclination of modern policy making mechanisms for shortterm perspectives and symbolic rather than substantial actions. At the international level, these factors are exponentiated by the lack of any international regulatory framework that would reduce and channel the blind powers at work among interested states and organisations willing to engage or contribute. The promising evolutions in the development and security communities call for more attention and political will for improvements to tackle the challenges of the securitydevelopment nexus in the capitals and strategic international fora.

\title{
Comprehensive Approach in zivil-militärischen Einsätzen - (k)ein Mehrwert in Afghanistan?
}

\author{
Markus Gauster*
}

\begin{abstract}
The international debate on civil-military interaction and coordination efforts in international crisis management has increased substantially since the beginning of the military intervention in Afghanistan in 2001 . Only by 2006 had it become clear that lacking "success stories" made international organisations, states and especially military actors ambitious with regard to comprehensive approaches (CA). The basic idea behind CA is to include most of the relevant actors at different levels in a structural manner to improve the impact of external engagement. However, the impact of this new strategy has not yielded sufficient results so far on the ground and appears hard to implement. At least the interaction between civilian, military and local components and the division of tasks can potentially be improved by increasing and improving communication. Instruments to achieve synergy effects from planning to implementation are at an early stage and the "peace dividend" as an added value for the local population is still missing. The political, conceptual and practical obstacles to implement CA are enormous. This article examines various levels of interaction, civil-military interfaces, indicators and the respective outcome derived from the engagement in Afghanistan in order to propose an analysis model for CA. This could contribute to the feasibility of future innovative CA concepts with regard to other conflict scenarios.
\end{abstract}

Keywords: Afghanistan, comprehensive approach, whole of government approach, unity of efforts Afghanistan, umfassender Ansatz, Whole-of-Government-Ansatz, Kohärenz

\section{Comprehensive Approach: Hintergrund und Untersuchungsebenen}

$\mathrm{E}$ in Comprehensive Approach (CA) kann als Leitphilosophie, Leitgedanke und Konzept für ein gemeinschaftliches Bemühen um eine koordinierte, komplementäre und kohärente Vorgangsweise im Rahmen des Internationalen Krisen- und Konfliktmanagements (IKKM) gesehen werden. ${ }^{1}$ Als Konzept ist ein CA auf der Ebene internationaler Organisationen (IOs) angesiedelt, weist eine deutliche NATOLastigkeit auf und wurde auch in diesem Umfeld geprägt. So ist durch den Comprehensive Approach Action Plan der NATO seit

* Markus Gauster ist wissenschaftlicher Mitarbeiter am Institut für Friedenssicherung und Konfliktmanagement (IFK) der Landesverteidigungsakademie in Wien. Aufenthalte in Afghanistan u. a. als Langzeit-Wahlbeobachter für die EU. Email: markus.gauster@bmlvs.gv.at.

1 Vgl. Barnet, Günther/Braumandl-Dujardin, Wolfgang: Ein Comprehensive Approach für Österreichs Beitrag zum internationalen Konflikt- und Krisenmanagement - ein Begriff, viele Möglichkeiten. In: Österreichische Militärische Zeitschrift (ÖMZ) 4/2011, S. $456 f$.
2008 die Richtung für einen CA in den Mitgliedstaaten auf der Basis gemeinsamer Planung, gemeinsamer Trainingsaktivitäten, strategischer Kommunikation und verstärkter Netzwerkbildung vorgegeben. ${ }^{2}$

Die sicherheitspolitische Debatte über CA-Ansätze in Afghanistan ist vielfältig, unübersichtlich und widersprüchlich zugleich. Sie impliziert unterschiedliche Begriffsverständnisse, Anwendungen, Ebenen und Kritiken. Sowohl in politischstrategischen Planungsprozessen als auch im operativen zivil-militärischen Einsatz kommt es zu einer Vermischung von Interaktionsebenen bei gleichzeitig mangelnder Abstimmung. Dies bringt oft Schwierigkeiten bei organisatorischen Abläufen mit sich. Wesentliche Stolpersteine ergeben sich auch durch Unterschiede in den jeweiligen Mandaten, Operationsplänen, Denkweisen sowie die geostrategische Relevanz des Einsatzraumes. Hinzu kommt, dass die Übernahme der CA-Idee auf

2 Vgl. http://www.nato.int/cps/en/natolive/topics_51633.htm (abgerufen am 12.9.2011). 
unterschiedlichen Ebenen komplexe Umsetzungsschritte erforderlich macht. ${ }^{3}$

„Koordination“ und „Kooperation“ involvierter Akteure waren bereits bei der Etablierung der International Security Assistance Force (ISAF) und der United Nations Assistance Mission in Afghanistan (UNAMA) 2001/02 Schlagworte, die - von der politischen Ebene ausgehend - auch in den entsprechenden Mandaten verankert wurden. ${ }^{4}$ Provincial Reconstruction Teams (PRTs) als Instrument strukturierter Interaktion zwischen Diplomatie, Militär und Entwicklungspolitik verstärkten seit 2004 Debatten über umfassende Ansätze - ausgehend vom Einsatzraum. ${ }^{5}$ Die NATO hat Begriffe bzw. Konzepte wie Unity of Effort und Effect-based Approach im Kontext integrierter und kohärenter Vorgehensweise in Afghanistan geprägt, nachdem sich die Sicherheitslage seit 2005 deutlich verschlechtert hatte. Da es auf internationaler Ebene wie auch auf der Ebene der Nationalstaaten kaum jemals den echten politischen Willen gab, einen CA als Konzept ernsthaft umzusetzen, hat sich die NATO auf dessen Implementierung auf militärischer Ebene versucht. Afghanistan wurde faktisch zum Erfahrungshintergrund, durch den sich die CA-Debatte in der NATO und die Debatte über Vernetzte Sicherheit in Deutschland überhaupt erst intensivieren konnten. Die negativen Entwicklungen in Afghanistan stellten somit die Problematik dar und der CA den Versuch einer unmittelbar aus und mit Bezug zu dieser Erfahrung entwickelten Lösung.

Die Umsetzung eines CA hängt vom Willen der politischen Akteure auf internationaler Ebene ab. Ein CA ist jedoch kein Selbstzweck - die direkten Auswirkungen sollten auf lokaler Ebene im Einsatzraum positiv zum Tragen kommen. ${ }^{6}$ Innerhalb dieser Bandbreite werden vom Autor fünf Strukturierungsmarken bzw. Untersuchungsebenen dargelegt, die auf einem CA als Leitgedanken basieren und in einem Analyseraster verarbeitet wurden. Die darin enthaltenen Faktoren bzw. Indikatoren könnten im Sinne einer verbesserten Planung, Durchführung und Evaluierung zukünftiger Missionen von Relevanz sein. So sind Erfahrungswerte aus dem IKKM in Afghanistan beispielsweise bereits im beträchtlichen Maße in die Operation EUFOR Tchad/RCA eingeflossen (z.B. Prinzipien des Last Resort, zivil dominiertes Projektmanagement, etc.), wodurch einige Fehler bzw. Zielkonflikte vermieden werden konnten. ${ }^{7}$ Auf mehrere, den Ebenen beigeordnete Faktoren im Analyseraster wird in der Folge eingegangen. ${ }^{8}$

3 Vgl. Barnet/Braumandl-Dujardin 2011.

4 Vgl. UNSCR 1817 (2008)

5 Vgl. Gauster, Markus: Provincial Reconstruction Teams in Afghanistan. An innovative instrument of international crisis management being put to the test. Marshall Center Occasional Paper Series No. 16, Garmisch-Partenkirchen 2008.

6 Vgl. Braumandl-Dujardin, Wolfgang/Feichtinger, Walter/Gauster, Markus: Comprehensive Approach: Vom strategischen Leitgedanken zur vernetzten Politik. Schriftenreihe der Landesverteidigungsakademie 8/2011, S. 19 ff.

7 Vgl. Gauster, Markus: Richtlinien und Wirkung zivil-militärischer Interaktion. Theorie und praktische Erfahrungen am Beispiel EUFOR Tchad/RCA. Schriftenreihe der Landesverteidigungsakademie 5/2010. Wien 2010, S. $90 \mathrm{ff}$.

8 Eine Fallstudie zu Afghanistan auf Basis des Analyserasters würde den vorgegebenen Rahmen sprengen. Daher erfolgt eine Untersuchung von Interaktionsebenen eines CA auf der Grundlage vorhandener Erfahrungswerte. Anzuführen ist, dass sich über die verwendeten Begrifflichkeiten bzw. Untersuchungsebenen in der internationalen CA-Debatte noch keine allgemein anerkannte Definition etabliert hat.
Tabelle: Analyseraster für Comprehensive Approach

\begin{tabular}{|c|c|}
\hline Untersuchungsebene & \begin{tabular}{|l} 
Prüfung folgender Faktoren \\
(keine taxative Aufzählung)
\end{tabular} \\
\hline 1. CA als Leitgedanke & $\begin{array}{l}\text { Politischer Wille für CA; Abstim- } \\
\text { mung und Harmonisierung der } \\
\text { Missionsziele; Anwendbarkeit von } \\
\text { Prinzipien des IKKM; Akzeptanz } \\
\text { des Einsatzes in den Bevölke- } \\
\text { rungen }\end{array}$ \\
\hline $\begin{array}{l}\text { 2. Whole of System } \\
\text { Approach/WoSA } \\
\text { (Ebene der IOs) }\end{array}$ & $\begin{array}{l}\text { Ambitionen; Machtprojektion; } \\
\text { Strategiebildung; Mandatslage; } \\
\text { IO-übergreifende Gremien; Füh- } \\
\text { rungspersönlichkeiten; Stärken } \\
\text { und Schwächen der IOs; Lessons- } \\
\text { Learnt-Prozesse; Zeitfaktor } \\
\end{array}$ \\
\hline $\begin{array}{l}\text { 3. Whole of Govern- } \\
\text { ment Approach/WoGA } \\
\text { (staatliche Akteure) }\end{array}$ & $\begin{array}{l}\text { Ambition des Staates; Konzept- } \\
\text { landschaft; rechtliche Rahmen- } \\
\text { bedingungen; Rolle des Militärs; } \\
\text { Entscheidungsprozesse; Koordina- } \\
\text { tions-, Steuerungs- und Finanzie- } \\
\text { rungsinstrumente }\end{array}$ \\
\hline $\begin{array}{l}\text { 4. Whole of Nation } \\
\text { Approach/WoNA (Staat, } \\
\text { Zivilgesellschaft) }\end{array}$ & $\begin{array}{l}\text { Grad des gesamtstaatlichen } \\
\text { Denkens; Akteurskonstellation; } \\
\text { Initiativen; Akzeptanz des Militärs } \\
\text { in der Zivilgesellschaft; NGO- } \\
\text { Ansätze; Schnittstellenfähigkeit; } \\
\text { gemeinsame Lagebeurteilung }\end{array}$ \\
\hline $\begin{array}{l}\text { 5. Lokaler CA } \\
\text { (im Einsatzraum bzw. in } \\
\text { der Krisenregion) }\end{array}$ & $\begin{array}{l}\text { Sicherheitslage; internationale } \\
\text { und lokale Akteurskonstellation; } \\
\text { Ambitionen und Kooperationsbe- } \\
\text { reitschaft; Grad der Abhängigkeit } \\
\text { von Akteuren untereinander; } \\
\text { Aufgaben- und Projektverteilung; } \\
\text { Cluster-Ansätze; Schnittstellen für } \\
\text { Interaktion }\end{array}$ \\
\hline
\end{tabular}

\section{Ebene Eins: Comprehensive Approach als strategischer Leitgedanke}

Die CA-Philosophie wird seit ca. 2006 zunehmend in Dokumenten, Policy- und Strategiepapieren verwendet und dadurch oft als „strategischer Imperativ“ für ein verbessertes IKKM gesehen. Der 3C-Ansatz (coordinated, coherent, complementary) erkennt z.B. die Relevanz zivilgesellschaftlicher Kräfte als Akteure im IKKM und versucht, den Dialog mit ihnen im Sinne der CAPhilosophie zu fördern. ${ }^{9}$ Dies entspricht auch dem Umstand, dass der Konflikt in Afghanistan sicherheits-, gesellschafts-, kultur- und wirtschaftspolitische Dimensionen aufweist, in denen „Dialog“ eine wesentliche Rolle spielt. Will man einen CA als eine strategische Übereinkunft für mehr Politikkohärenz zur Bewältigung eines komplexen Szenarios wie Afghanistan begreifen, stellen sich Fragen der Abstimmung bzw. Harmo-

9 Vgl. Feichtinger, Walter/Werther-Pietsch, Ursula/Barnet, Günther: Koordiniert, komplementär und kohärent agieren in fragilen Situationen - die Wiener 3C-Konferenz. Schriftenreihe der Landesverteidigungsakademie Nr. 12/2010/S, Wien 2010. 
nisierung politischer Ziele, des strategischen Vorgehens, der Prioritätensetzung, der Umsetzbarkeit, etc.

In diesem Kontext kann insbesondere im Zuge der deutschen Debatte über vernetzte Sicherheit eine „Afghanisierung“ der deutschen Sicherheitspolitik konstatiert werden. Afghanistan stellt in Verbindung mit der CA-Philosophie einen strategischen Leitgedanken für Sicherheitspolitik und Militär dar. Konzepte sind auf den Afghanistan-Einsatz abgestimmt und dominieren die übergeordnete sicherheitspolitische Agenda. Insbesondere das Verteidigungsressort, das unter großem (Erfolgs-) Druck steht, stellt koordiniertes zivil-militärisches Vorgehen und einen umfassenden Ansatz in den Vordergrund. Insbesondere Akteure der Zivilgesellschaft stehen hingegen der Realisierung vernetzter Sicherheit zunehmend kritisch gegenüber. ${ }^{10}$

Die Umsetzung eines CA in Afghanistan kann u.a. anhand der faktischen Aufgabenteilung zwischen militärischen, polizeilichen, diplomatischen, humanitären und entwicklungsbezogenen Akteuren untersucht werden. Eine Etablierung der von diesen akzeptierten Schnittstellen zur Abstimmung des Vorgehens der Akteure erweist sich jedoch als äußerst problematisch. Die Strategien zur Bearbeitung der Herausforderungen in Afghanistan stellen sich im Kontext umfassender Ansätze als sehr diffus dar. So existieren z.B. Counterinsurgency-Ansätze, die von Staat zu Staat unterschiedlich ausgelegt werden, oder Operationspläne der ISAF und der Operation Enduring Freedom parallel zu Entwicklungsstrategien wie der Afghanistan National Development Strategy, Strategiepapieren der Entsendestaaten oder Mandaten der UNO. Dazu kommt das Dilemma für das Militär, sowohl einen bevölkerungszentrierten Ansatz umzusetzen und Zivilisten zu schützen, zugleich aber auch Aufstandsbekämpfung zu betreiben. ${ }^{11}$ Eine Abstimmung der Einzelstrategien im Sinne eines koordinierten Vorgehens ist in der Realität kaum machbar bzw. nicht gewollt.

\section{Ebene Zwei: Whole of System Approach am Beispiel der UNO, EU und NATO in Afgha- nistan}

Abgeleitet vom strategischen Leitmotiv CA stellt der Whole of System Approach (WoSA) die oberste Koordinierungsebene im CA-Spektrum dar. Dabei geht es insbesondere um die Abstimmung von IOs untereinander im Sinne einer Systemhierarchie. In Afghanistan bezieht sich ein WoSA somit auf das koordinierte Vorgehen der NATO, EU und der UNO sowie einer Reihe von weiteren IOs (z. B. Weltbank, Rotes Kreuz, etc.). Auch die Einbindung nichtstaatlicher Akteure spielt dabei eine Rolle, um im idealtypischen Fall ein Zusammenwirken aller wesentlichen Akteure zu erreichen.

10 Dieser Umstand ergibt sich vor allem daraus, dass die Grenzen zwischen zivilem und militärischem Engagement aufgeweicht wurden, da das Militär (ISAF) zunehmend auch „zivile“ Aufgaben abseits militärischer Kernaufgaben übernahm. Das trug dazu bei, dass sich die Arbeitsbedingungen im humanitären Umfeld für viele NGOs deutlich verschlechterten.

11 Allard Wagemaker (Netherlands Defence Academy) im Gespräch mit dem Autor, 25.7.2011.

\subsection{Die UNO im Whole of System Approach}

Seitens der UNO wird das Kohärenzprinzip im Sinne eines WoSA in mehreren Dokumenten zu Afghanistan eingefordert. Die UN-Sicherheitsratsresolution (UNSCR) 1890 (2009) betont die Notwendigkeit eines Comprehensive Framework im Sicherheitssektor; in der UNSCR 1868 (2009) wird auf die Bedeutung einer umfassenden Vorgehensweise hingewiesen; in den UNSCR 1817 (2008) und 1974 (2011) wird eine Verbesserung der Koordination gefordert, um kriminelle Aktivitäten und die Drogenökonomie zu bekämpfen. Die Ziele bzw. die Forderungen in den UN-Mandaten im Hinblick auf einen CA sind hoch. Am Beispiel der UNAMA mit ihrem Anspruch auf Koordination von Akteuren ist jedoch der generell schwache Status der UNO in der Region zu erkennen. Auch wird UNAMA oft als nicht neutral gesehen und damit nicht als Koordinator akzeptiert. ${ }^{12}$ ISAF und die meisten Entsendestaaten bevorzugen den direkten bzw. bilateralen Zugang zum Einsatzraum und sind nicht Willens, sich gleichsam UN-Strukturen unterzuordnen.

Die Schnittstellenfrage zur Verknüpfung unterschiedlicher Ebenen stellt einen kritischen Faktor im Rahmen eines WoSA dar. Neutralität und Unparteilichkeit, aber auch politische Akzeptanz sind wesentliche Voraussetzungen für einen Koordinierungsanspruch bzw. eine Schnittstellenfähigkeit. UNAMA konnte diese Voraussetzungen nicht erfüllen und war in ihrer Funktion politisch von niemandem gewollt, was sich besonders nach den von massiven Wahlfälschungen überschatteten Präsidentschaftswahlen 2009 zeigte. ${ }^{13}$ Daraus sind ein Kernproblem und gleichzeitig ein Dilemma abzuleiten. IOs können per se nicht neutral bzw. unparteiisch sein und sind somit als Koordinierungsstellen kaum geeignet. Nationale Vorbehalte tragen ebenfalls zu diesem Dilemma bei. Für militärische Akteure stehen diverse COIN-Strategien im Mittelpunkt, die wiederum viele zivile Akteure als unvereinbar mit humanitären Ansätzen und Entwicklungsprinzipien berachten, da sie in der Regel nicht auf lokalem Konsens beruhen. ${ }^{14}$ Ein WoSA ist in Afghanistan weder durch die UNO noch durch andere Akteure realisierbar. Man kann aber auch argumentieren, dass die in UN-Dokumenten angeführte Dringlichkeit kohärenten Vorgehens von IOs und Staaten zumindest für eine Annäherung von Positionen und zu verstärktem zivil-militärischem Dialog geführt hat.

\subsection{Die EU und NATO im Whole of System Approach}

Seitens der EU und der NATO können divergierende politische Ansichten, nationale Vorbehalte und die angespannte Budgetsituation der jeweiligen Mitgliedstaaten als Hindernisse für komplementäres Vorgehen gesehen werden. ${ }^{15}$ Auch die zahlreichen Sonderbeauftragten bzw. zivilen Repräsentanten

12 Stefan van Laar (NGO HealthNet TPO) im Gespräch mit dem Autor, 10.5.2010.

13 Vgl. Margesson, Rhoda: UNAMA: Background and Policy Issues. CRS Report for Congress, Washington 2010. S. 2.

14 Karl Torring (NGO Swedish Committee for Afghanistan) im Gespräch mit dem Autor. Wien, 5.5.2011.

15 Vgl. Jakobsen, Peter Viggo: "NATO‘s Comprehensive Approach to Crisis Response Operations: A Work in Slow Progress." Kopenhagen 2008, S. $41 \mathrm{ff}$. 
für Afghanistan, ob von der EU, NATO, UNO oder einzelnen Entsendestaaten, verfügen kaum über Handlungsspielräume ${ }^{16}$ und erscheinen daher nicht als Schnittstelle geeignet. Weitgehend akzeptierte Führungspersönlichkeiten (wie es z.B. Lakhdar Brahimi 2001 war) fehlen. Somit stellt sich die Frage, welche politische Gewichtung der CA im Rahmen des Engagements der EU in Afghanistan aufweist. Dies lässt sich im Bereich Polizeiaufbau beleuchten, der die wenig abgestimmte Vorgangsweise der EU und NATO in Afghanistan aufzeigt. So führte das weder personell noch finanziell ausreichend ausgestattete Engagement der EU im Rahmen des Polizeiaufbaus von EUPOL Afghanistan ${ }^{17} \mathrm{zu}$ einer graduellen Übernahme von Agenden durch die USA und in der Folge 2009 zur Gründung der NATO Training Mission-Afghanistan (NTM-A).

Seit der Etablierung der NTM-A stehen sich zwei PolizeiaufbauAnsätze gegenüber: Der auf schnelle Ausbildung orientierte, militärische Ansatz der USA und ein längerfristig orientierter EU-Ansatz zur Unterstützung des Aufbaus ziviler Polizeistrukturen. Es kann argumentiert werden, dass das notwendige Vertrauen der Bevölkerung in die afghanische Polizei durch den EU-Ansatz eher gestärkt werden könnte als durch das kurze Ausbildungsprogramm der NTM-A. ${ }^{18}$ Die EU leidet allerdings unter Ressourcenmangel und politischen Unstimmigkeiten - EUPOL wird als rein „politische Mission“ angesehen.

Diese Faktoren haben ambivalente Auswirkungen auf Aufbau, Einsatzbereitschaft und Legitimität der afghanischen Polizei. So hat die afghanische Polizei mehr Opfer in der Aufstandsbekämpfung zu beklagen als das afghanische Militär, was die militärische Komponente der Polizeiausbildung durchaus relevant macht. ${ }^{19}$ Insbesondere muss ISAF oft die Polizei schützen, ohne ihre Aufgaben zu übernehmen. ${ }^{20}$ Dennoch erscheint es wichtig, auch den zivilen Ausbildungsschwerpunkt (z.B. Polizeiausbildung durch zivile Polizeikräfte) zu betonen. Somit ist zu konstatieren, dass die unterschiedlichen Konzepte der Polizeiausbildung zu divergierenden Maßnahmen und Resultaten geführt haben, die einem WoSA zuwiderlaufen. Ein komplementäres Vorgehen von EU und NATO lässt sich aus der unterschiedlichen Vorgehensweise und der Lage 2011 nicht ableiten.

\section{Ebene Drei: Whole of Government Approach - ausgewählte Problemkreise und Erfahrungs- werte}

Auf der Ebene des WoGA sollen staatliche Akteure für die internationale Konfliktbearbeitung erfasst, Maßnahmen gebündelt und inter- wie intraministeriell koordiniert werden. Im WoGA legt ein Staat über seine Ressorts und Einrichtungen als

16 Anja Opitz im Gespräch mit dem Autor, 29.6.2011.

17 Vgl. <http://www.isis-europe.org/pdf/July2011EUPOLAFGHANISTAN.pdf

18 Vgl. Bayley, David H.: Police Reform as Foreign Policy. In: The Australian and New Zealand Journal of Criminology, Vol. 38, No. 2, 2005, 206-215, sowie Bayley, David H./Perito, Robert M.: The Police in War: Fighting Insurgency, Terrorism, and Violent Crime, Lynne Rienner, Boulder 2010.

1960 Prozent aller getöteten afghanischen Sicherheitskräfte seit 2007 waren Polizisten. Vgl. Schmidt, Martin: Ausbildung der Afghanischen Nationalen Polizei (ANP) - Aufstandsbekämpfung oder Aufbau einer zivilen Polizei? In: SIAK-Journal, Wien 2011.

20 Michael Daxner im Gespräch mit dem Autor, 29.6.2011.
Beitragsleister für externes Engagement fest, was er im Kontext seiner Ambitionen zur Verfügung stellen kann. Voraussetzung für gemeinsame Planung und kohärentes Vorgehen ist Klarheit über die jeweiligen Ziele der in Frage kommenden staatlichen Akteure im Entsendestaat. Hindernisse sind die oftmals sehr unterschiedlichen Handlungslogiken und Selbstverständnisse einzelner Ressorts sowie rechtliche Limitierungen (z.B. das deutsche Ressortprinzip). In diesem Zusammenhang gilt es eine Balance zu finden und die Zugeständnisse der Akteure in einem für sie tragbaren Rahmen zu halten. ${ }^{21}$

Die größten Gegensätze finden sich naturgemäß zwischen staatlichen und nichtstaatlichen Akteuren. Für staatliche Akteure, insbesondere für das Militär, ist es in EU-Staaten opportun, über den Sinn oder Zweck einer übergeordneten Strategie zu diskutieren. Für NGOs vor Ort, die gemäß ihrer eigenen Strategie vorgehen, stehen politische Diskussionen nicht im Mittelpunkt. Nichtstaatliche Akteure sind bei zivil-militärischen Konzepten mit - für sie fremden - bürokratischen Abläufen konfrontiert. Gelingt es hier ein Gleichgewicht zu finden, sind die unterschiedlichen Herangehensweisen ein Gewinn. ${ }^{22}$ Gegenseitige Toleranz stellt damit eine essentielle Grundlage nationaler und internationaler zivil-militärischer bzw. umfassender Ansätze dar.

Eine wesentliche Aufgabe von Steering Groups als staatliche Steuerungsinstrumente, die z.B. Großbritannien oder Dänemark im Zuge des Afghanistan-Einsatzes eingerichtet haben, ist es, Strategien zur Interaktion der einzelnen Akteure auf WoGAEbene zu entwickeln. Mit entsprechender Finanzkraft ausgestattet, stellen Steering Groups ein wesentliches Element zur Steuerung staatlicher Akteure und Entwicklung eines WoGA dar. ${ }^{23}$ Eine Zusammenführung von Kompetenzen sollte sich gleichsam in Form einer institutionalisierten Schnittstelle niederschlagen. Informelle Netzwerke, Kanäle oder Treffen sind jedoch weiterhin eine unverzichtbare Basis für abgestimmtes Vorgehen.

Die Vernetzung einzelner staatlicher Akteure beginnt insbesondere bei der Finanzierung gemeinsamer Projekte. Ein innovativer Ansatz ist die Einrichtung von Pools, die Finanzierungsmittel für gemeinsame Projekte und Initiativen darstellen. Die Idee dahinter ist, dass Zusammenarbeit mit zusätzlichen Geldmitteln belohnt wird. Der Europäische Entwicklungsfonds kann z.B. mit Hilfe seiner Transferleistungen zivilgesellschaftliche Akteure zusammenführen. Auch in Großbritannien werden Pools auf unterschiedlichsten Ebenen eingesetzt. So wird auch die Entwicklung von gemeinsamen Strategien gefördert. Pools sind jedoch nur ein kleiner Teil des bilateralen finanziellen Engagements Großbritanniens im jeweiligen Bereich. Es besteht außerdem die Gefahr, dass die Pools durch die einzelnen Akteure geleert werden - ein einfacher Weg, um finanzielle Mittel aufzustocken. ${ }^{24}$ Gemeinsame Finanzierungsstrukturen

21 Hans-Georg Ehrhart in einem Vortrag an der Evangelischen Akademie Loccum, 5.12.2010.

22 Schaper, Marcus: Entwicklungshilfe mit Stahlhelm? Vernetzte zivile und militärische Planung für Frieden und Stabilität in Krisengebieten. Bericht zur Tagung an der Evangelischen Akademie Loccum / D, 3.-5.12.2010, S. 6.

23 Finn Stepputat in einem Vortrag an der Evangelischen Akademie Loccum, 4.12.2010.

24 Daniel Korski in einem Vortrag in Loccum, 4.12.2010. 
können daher ein Antrieb für kohärentes Vorgehen auf allen CA-Ebenen sein.

Vieles, was Interaktionsmechanismen betrifft, geht auf Erfahrungen aus der Arbeit vor Ort zurück. Das „institutionelle Gedächtnis“ bleibt jedoch in vielen Fällen beschränkt und Gelerntes wird oft nicht weitergegeben, was für das IKKM über Afghanistan hinaus ein Problem darstellt. Unterschiedliche WoGAs führten zwar zu mehr Interaktion auf staatlicher Ebene; für Afghanistan konnte dieser Umstand jedoch kaum einen erkennbaren Mehrwert in punkto Sicherheit und Wiederaufbau bringen.

\section{Ebene Vier: Whole of Nation Approach}

\subsection{Allgemeine Aspekte}

In einem WoNA sollen - ausgehend von einem WoGA - auch nichtstaatliche Akteure wie zivile Hilfsorganisationen für Maßnahmen des IKKM eingebunden werden. NGOs haben damit für Staaten und IOs im IKKM an Relevanz gewonnen. „Vernetzte Sicherheit" steht dem WoNA als Konzept nahe, wenngleich es bisher nicht ausreichend präzise definiert wurde. Die Interaktion bzw. der Dialog soll damit gefördert werden, weil dadurch (zumindest nach Ansicht von Staaten und IOs) Effektivität und Effizienz der Missionen gesteigert werden können. Eine intensive zivil-militärisch geprägte Vorbereitung auf Einsätze stellt einen Eckpfeiler des WoNA dar.

\subsection{WoNA am Beispiel der Niederlande}

Im Fall des Engagements der Niederlande mit ihrer Präsenz in der afghanischen Provinz Uruzgan 2006-2010 wurden im Sinne eines WoNA im Vorhinein die Voraussetzungen für eine Kooperation mit niederländischen NGOs hergestellt. Diese operierten in der Regel bereits lange vor dem militärischen Einsatz in Uruzgan, was sich als vorteilhaft für eine abgestimmte Strategie erwies. Die Lage im Einsatzgebiet wurde durch das Außenministerium, das Verteidigungsministerium und NGOs (u.a. HealthNet TPO) gemeinsam bewertet, um einen Rahmen für die wichtigsten Aktivitäten zu schaffen. Dies hat sich in der Praxis als sehr nützlich erwiesen. ${ }^{25}$

Das niederländische Engagement verfolgte in Uruzgan einen bevölkerungszentrierten bzw. kontextbezogenen Ansatz. Die Akteure konnten sich jedoch während der gesamten Einsatzzeit auf keine klare (umfassende) Policy seitens der niederländischen Regierung stützen. Zudem befanden sich die niederländischen Kräfte im Konflikt mit dem eher feindzentrierten US-Ansatz des ISAF-Regionalkommandos Süd. ${ }^{26}$ Dazu kam, dass in Konzepten, im Operationsplan der ISAF oder auf der Ebene des Regionalkommandos kaum Direktiven bezüglich eines kohärenten Vorgehens existierten, was sich bei der Interaktion

25 Van der Gaag-Halbertsma, Jet/de Vries, Hugo/Hogveen, Bart: Civil-Military Cooperation from a 3-D Perspective, in: Rietjens, Sebastiaan/Bollen, Myriame: Managing Civil-Military Cooperation: A 24/7 Joint Effort for Stability. Aldershot 2008, S. 40.

26 Allard Wagemaker im Gespräch mit dem Autor, 12.7.2011. im Feld widerspiegelte. Es gab keinen offiziellen 3D-Ansatz im Sinne einer strukturellen Verschränkung der Komponenten Diplomacy, Defence und Development und damit auch keinen echten WoNA. Vielmehr wurde das Bild eines WoNA von den Medien in den Niederlanden geschaffen bzw. nach kanadischem Vorbild kopiert. ${ }^{27}$ Dies wird auch dadurch bestätigt, dass ein 3D-Konzept (wie ein WoNA) klare koordinative Vorgaben braucht, niederländische Akteure sich jedoch nicht koordinieren ließen - somit konnte ein 3D-Ansatz im Feld nicht zur Anwendung kommen. ${ }^{28}$

Grundsätzlich wurde die niederländische CA-Debatte im Kontext Afghanistans stark politisiert. Nach Ansicht niederländischer NGO-Vertreter sind dominierende politische Eigeninteressen einzelner Ressorts wichtiger als konkrete humanitäre Hilfe vor Ort. ${ }^{29}$ Im Sinne eines komplementären gesamtstaatlichen Vorgehens ist daher die Politik gefordert, die Voraussetzungen für umfassendes Vorgehen bei Auslandseinsätzen zu schaffen. Das Militär stellt dabei nur einen (von mehreren) Erfüllungsgehilfen zur Umsetzung politischer Ziele dar. ${ }^{30}$ Eine „Gesamtstrategie“ im Sinne eines WoNA war seitens der niederländischen Regierung für Afghanistan jedoch nicht vorhanden. Vielmehr waren separate Strategien des Militärs, der Diplomatie und der Entwicklungspolitik maßgebend. Dabei hat das Militär keine politisch-strategischen Vorgaben in Afghanistan umgesetzt, sondern ging weitgehend improvisierend und situationsabhängig vor („Ad-hocracy“ im Feld). ${ }^{31}$

\section{Ebene Fünf: Aspekte der Interaktion auf lokaler Ebene}

Definiert man das CA-Konzept auf lokaler (operativ-taktischer) Ebene durch die Abstimmung eines gemeinschaftlich definierten Zieles relevanter Akteure, so ist das Konzept in Afghanistan gescheitert. Entsprechende Abstimmungsprozesse erfassen nur einen geringen Teil der Akteure, von denen die meisten - staatliche wie nichtstaatliche - jedoch eigene Interessen verfolgen. Dies liegt vor allem daran, dass politische Befindlichkeiten, Vorbehalte, Bürokratie, ad hoc geprägtes Vorgehen sowie divergierende Standards und Ansichten den operativen Handlungs- und Interaktionsspielraum von Akteuren in Afghanistan bestimmen und gleichzeitig beschneiden. Trotzdem ist eine Annäherung zivil-militärischer Positionen im Sinne einer Aufgabenteilung wohl am ehesten auf lokaler Ebene realistisch, da die Bildung von Zweckgemeinschaften Synergieeffekte erzeugen kann (Beispiel Niederlande).

Oft erschweren unrealistische Erwartungshaltungen militärischer und ziviler Akteure sowie Konkurrenzdenken ein kohärentes und komplementäres Vorgehen. In der Einsatzpraxis zeigte sich, dass der stärkste Akteur auch den größten Einfluss auf das Geschehen hat. So waren viele Aktivitäten der Niederlande in Uruzgan vom Militär dominiert, das quantitativ den

27 Stefan van Laar (NGO HealthNet TPO), im Interview mit dem Autor, 18.10.2010.

28 Albert van Hal (NGO Cordaid), im Gespräch mit dem Autor, 19.10.2010.

29 Van Laar, 18.10.2010.

30 Jair van der Lijn im Gespräch mit dem Autor, 20.10.2010.

31 Lenny Hazelbag (Netherlands Defence Academy) im Gespräch mit dem Autor, 22.10.2010. 
zivilen Akteuren überlegen war. Diplomaten der Niederlande hatten nur wenig Entscheidungskompetenz, da sie nicht kontextspezifisch ausgebildet waren, kaum über finanzielle Mittel verfügten und in der Minderzahl waren. ${ }^{32}$ Trotzdem wurden Diplomaten auch als Unterstützer einer niederländischen Gesamtagenda in Afghanistan gesehen. ${ }^{33}$ Humanitäre Hilfe und Entwicklungszusammenarbeit durch die NGOs fanden im Einsatzraum verstärkt Eingang, weil ausreichende finanzielle Unterstützung seitens der niederländischen Regierung vorhanden war. ${ }^{34}$

Eine Institutionalisierung der lokalen Interaktion mit dem Militär ist aus Sicht von NGOs grundsätzlich abzulehnen, da diese gegen humanitäre NGO-Prinzipien verstoßen würde. Der Fall der schwedischen NGO Swedish Committee for Afghanistan (SCA), die fast ausschließlich mit lokalen Mitarbeitern operiert, zeigt zudem, dass die Anwendung des Local-Ownership-Prinzips nicht mit einem CA vereinbar ist, da ein Freiraum für die Verfolgung der eigenen (NGO-) Agenda unverzichtbar ist. ${ }^{35}$ Die ersatzweise Übernahme des sehr spezifischen Aufgabenspektrums einer NGO wie SCA (Ausbildung und Training von Lehrpersonal, Gesundheit, landwirtschaftliche Entwicklung, etc.) durch das Militär in vergleichbaren Szenarien erscheint unrealistisch und zeigt Grenzen der Interaktion auf. ${ }^{36}$

\section{Erkenntnisse aus dem Afghanistan-Einsatz im Kontext des Comprehensive Approach}

Wenn man die Realität im Einsatzraum und im strategischen Umfeld betrachtet, konnte der CA-Leitgedanke in Form eines WoSA oder WoNA 2006-2011 nicht umgesetzt werden und daher auch nicht zu einer positiven Transformation des Konflikts beitragen. Stattdessen musste die NATO bittere Lehren ziehen und karge Ergebnisse verbuchen. Obwohl viele Akteure die Relevanz einer kohärenten Herangehensweise bekräftigen und bis zu einem gewissen Grad aufeinander angewiesen sind, dominierten Partikularinteressen vor Ort. Die Situation im Einsatzraum verschlechterte sich nicht zuletzt dadurch in den meisten Bereichen.

Es zeigt sich, dass zivilem Personal im IKKM mehr Relevanz und Befugnisse eingeräumt werden sollten, die gegenüber dem Militär durchsetzbar sind. Davon ausgehend erscheint es auch im Hinblick auf den NATO-Ansatz notwendig, zivile Kapazitäten im Sinne eines CA verstärkt einzubinden. ${ }^{37}$ Aspekte der Koordination und Aufteilung von Ressourcen für Einsätze sind im Vorhinein zu regeln und sollten von allen wesentlichen Akteuren getragen werden. Ein Anstoß hierzu kann nur von höchster politischer Ebene kommen und setzt institutionsübergreifenden Einfluss voraus. In Afghanistan wurde diesen Überlegungen seitens des IKKM keine übergeordnete Bedeutung beigemessen.

32 Van der Lijn, 20.10.2010

33 Wagemaker, 12.7.2011.

34 Van der Lijn, 20.10.2010.

35 Vgl. Braumandl-Dujardin/Feichtinger/Gauster 2011, S. 122.

36 Peter Brune (Swedish Committee for Afghanistan) im Gespräch mit dem Autor, 22.11.2010.

37 Vgl. Feichtinger, Walter: Internationales Konflikt- und Krisenmanagement. Grundprobleme und innovative Ansätze. In: Pucher, Johann/Frank, Johann (Hrsg.): Strategie und Sicherheit 2011. Globale Herausforderungen - Globale Antworten. Wien, Köln, Weimar 2011, S. 314.
Auf der Ebene des WoNA stellt sich die Frage, ob und inwieweit NGOs sich hier einbinden lassen und wenn ja, ob sich die Wirkung des IKKM dadurch verbessern lässt. Eines erscheint evident - IOs und staatliche Akteure erheben oft einen Koordinationsanspruch, jedoch wollen die wenigsten nichtstaatlichen Akteure „koordiniert werden“. Ein WoGA erscheint bis zu einem gewissen Grad machbar, da Ressorts zunehmend offener für Kooperationen sind (z.B. in Form der britischen Stabilisation Unit). Als Grenze der Machbarkeit ist der Übergang zwischen WoGA und WoNA anzusehen. Niederländische NGOs ließen sich in Uruzgan trotz staatlicher Subventionen nicht in eine militärische Clear-Hold-Build-Strategie einbinden. Damit bewahrten sie sich weitgehend ihre Unabhängigkeit und Freiräume im Sinne eines „WoNA light.“ Die zentralen NGOVorwürfe einer Gefährdung bzw. Behinderung ihrer Mitarbeiter bei der Arbeit durch militärische Präsenz konnten durch intensiven zivil-militärischen Dialog weitgehend ausgeräumt werden.

Die lokale Ebene stellt im Sinne der angeführten Faktoren im Analyseraster einen unterbewerteten Faktor im IKKM dar. ${ }^{38}$ Vielfach wird ignoriert, dass auch in Afghanistan lokale wirtschaftliche Aktivitäten in der einen oder anderen Form trotz kriegsähnlicher Zustände weitergehen. ${ }^{39}$ Konflikte um Ressourcen von Geberstaaten können dabei von externen Akteuren nicht einfach durch Präsenz und „Koordination“ gelöst werden. Vielmehr sollten lokale Akteure dazu gebracht werden, selbst zur Lösung beizutragen. Eine Option im CA-Kontext wäre daher, lokale Akteure nachhaltig einzubinden und ihnen die Möglichkeit zu geben, zur Lösung der Konfliktursachen beizutragen. Dies ist jedoch noch Zukunftsmusik, könnte aber dazu führen, dass sich gemeinsame Ziele und Visionen internationaler militärischer und ziviler Akteure in Abstimmung mit lokalen Strukturen entwickeln. In Afghanistan ist man davon noch weit entfernt, wenn man das Beispiel der zweifelhaften Kooperation von PRTs mit Milizenführern heranzieht. Einheimische Entscheidungsträger sind vom Militär jedenfalls in einen Dialog auf Augenhöhe einzubeziehen. Dies gilt auch für die Bevölkerung, weil diese letztendlich ein Hauptansprechpartner für das Militär ist, deren Gunst es zu gewinnen gilt.

\section{Fazit}

In der Einsatzrealität zeigte sich unter den Akteuren in Afghanistan ein multipler, auf mehreren Ebenen ablaufender Wettbewerb um Macht, Relevanz und Dominanz, der umfassendem Vorgehen zuwiderläuft und auch kaum zu überwinden ist. ${ }^{40}$ Nur Akteure, die plakative und vermarktbare „Ergebnisse“ vor-

38 Vgl. Freire, Maria Raquel et al., Mapping Research on European Peace Missions. Clingendael Security Paper Nr. 14. Netherlands Institute for International Relations Clingendael, Den Haag 2010, sowie Werther-Pietsch, Ursula: Sicherheit und Entwicklung. Zwei Pfeiler eines Systems? Schriftenreihe der Landesverteidigungsakademie 2/2009/S. Wien, Genf 2010.

39 Vgl. Gauster, Markus/Maruszczak, Markus: The Underestimated Relevance of Economic Impacts of Crisis Response Operations. In: Feichtinger, Walter/ Gauster, Markus/Tanner, Fred (Hrsg.): Economic Impacts of Crisis Response Operations. An Underestimated Factor in External Engagement. In: Schriftenreihe der Landesverteidigungsakademie 5/2010/S. Wien, Genf 2010, S. 12.

$40 \mathrm{Vgl}$. auch den Wettbewerb um Relevanz bei der UNO in: Afghanistan: UN Losing PR Battle in Kabul. http://www.eurasianet.org/node/63238. Eurasianet, 6.4.2011. 
weisen konnten, durften und dürfen weiterhin mit finanzieller Unterstützung rechnen. Der Grad der Interaktion von Akteuren in Afghanistan ist daher nach wie vor gering.

Der angekündigte Truppenrückzug aus Afghanistan bis 2014 führt eher weg von CA-Ansätzen. Ob Einzelstaaten der NATO oder der EU bei zukünftigen Einsätzen eine uneingeschränkte Führungsrolle bzw. einen Rückgriff auf benötigte nationale Ressourcen im Sinne eines funktionierenden CA zugestehen werden, darf - eingedenk der Erfahrungen aus Afghanistan und der Staatsschuldenkrisen in der EU - stark bezweifelt werden. Somit kann ein CA nur dann einen Mehrwert in der Einsatzpraxis bringen, wenn zentrale Aspekte einer geplanten Mission bereits im Vorfeld geklärt werden. Insbesondere betrifft das folgende Fragen: Wofür wird geplant, wer definiert den Endstatus, welche Koordinierungsstellen können vereinbartwerden, was sind die Hauptaufgaben des Militärs und welche Akteure stehen einem CA positiv bzw. negativ gegenüber?

Die Umsetzung eines CA in Afghanistan ist gescheitert. Die Uneindeutigkeit einer Vielzahl an vorhandenen Ansätzen und widersprüchlichen Handlungen hat zur aktuellen Lage beigetragen. Chancen für eine positive Wirkung des CA-Konzepts bestehen jedoch auf lokaler Ebene. Das IKKM hat sich im Hinblick auf WoGA und WoNA durch den Afghanistan-Einsatz weiterentwickelt und wird möglicherweise zur verbesserten Wirksamkeit in anderen Einsatzszenarien beitragen können. Angeführt werden kann hier vor allem die Aufnahme eines verstärkten Dialogs zwischen staatlichen, nichtstaatlichen und lokalen Akteuren. Die Schaffung eines gemeinsamen Verständnisses über politisch-strategische Ziele, die in Einsatzräumen verfolgt werden, erscheint unrealistisch.

\title{
Zur Bedeutung der Vernetzten Sicherheit - eine militärische Perspektive
}

\author{
Ralph Thiele*
}

\begin{abstract}
The Comprehensive Approach is the driving element in German security policy. It aims at achieving security by much more than solely military means and strives for an enhanced investment of scarce resources by their well orchestrated, synergetic application. Successful crisis management and conflict prevention do require the involved political, civilian and military actors to operate in a coherent and networked fashion. Discussing common strategies and objectives including their operational implementation in crisis management offers a unique opportunity to promote the Comprehensive Approach.
\end{abstract}

Keywords: German Armed Forces, Comprehensive Approach, Human Security, civil crisis management, peace-building Bundeswehr, Vernetzte Sicherheit, Menschliche Sicherheit, Zivile Krisenprävention, Friedenskonsolidierung

\section{Geschützte Freiheit}

$\mathrm{D}$ er Ansatz Vernetzter Sicherheit bezweckt, Sicherheit mit weitaus mehr als nur militärischen Mitteln zu gewährleisten. Sein Potenzial für einen wirklich integrativen, umfassenden Zugang zu Sicherheit, für mehr Kohärenz und Synergie und die damit verbundene Stärkung der zivilen Fähigkeiten wird allerdings häufig von entscheidenden Akteuren nicht gesehen. Auch deshalb gelingt bislang die überzeugende Umsetzung Vernetzter Sicherheit in der Planung und Durchführung von Einsätzen noch nicht.

Im Unterschied zu dem eher pragmatischen Verhältnis zwischen Nichtregierungsorganisationen und Streitkräften in anderen befreundeten Staaten haben in Deutschland viele zivile Akteure noch ein sehr überkommenes, teilweise ideologisches

* Oberst i.G. Ralph Thiele ist Vorsitzender der Politisch-Militärischen Gesell schaft Berlin.
Bild von Streitkräften. Der Ansatz der Vernetzten Sicherheit wird häufig als ein Versuch staatlicher Sicherheitspolitik gesehen, humanitäre Hilfe, Entwicklungs- und Friedenspolitik und ihre Akteure zu versicherheitlichen und dadurch zu vereinnahmen. So sehen die meisten Nichtregierungs- und Hilfsorganisationen die zivil-militärische Zusammenarbeit in Afghanistan sehr kritisch. Entsprechende Vorwürfe werden in Richtung Bundeswehr und NATO laut.

„Sicherheit ist geschützte Freiheit - nach innen und nach außen “, so hat es Bundesverteidigungsminister Thomas de Maizière formuliert. Gesellschaft, Werte und Normen sind zu schützen, ebenso Entwicklungen in anderen Politikbereichen wie Wirtschafts- und Finanzpolitik. Die traditionelle Unterscheidung zwischen äußerer und innerer Sicherheit verliert angesichts neuer Bedrohungen ihre Bedeutung. Selbstver-

1 Vgl. BMVg, Rede von Bundesverteidigungsminister Thomas de Maizière auf der 8. Handelsblattkonferenz am 25. Oktober 2011 in Berlin. 